\title{
Fredholm Modules for Quantum Euclidean Spheres
}

\author{
Eli Hawkins ${ }^{1}$, Giovanni Landi ${ }^{2}$ \\ ${ }^{1}$ Scuola Internazionale Superiore di Studi Avanzati \\ Via Beirut 4, I-34014 Trieste \\ mrmuon@mac.com \\ ${ }^{2}$ Dipartimento di Scienze Matematiche, Università di Trieste \\ Via Valerio 12/b, I-34127 Trieste \\ and INFN, Sezione di Napoli, Napoli \\ landi@univ.trieste.it
}

October 2002

\begin{abstract}
The quantum Euclidean spheres, $\mathrm{S}_{\mathrm{q}}^{\mathrm{N}-1}$, are (noncommutative) homogeneous spaces of quantum orthogonal groups, $\mathrm{SO}_{\mathrm{q}}(\mathrm{N})$. The $*$-algebra $A\left(S_{\mathrm{q}}^{\mathrm{N}-1}\right)$ of polynomial functions on each of these is given by generators and relations which can be expressed in terms of a self-adjoint, unipotent matrix. We explicitly construct complete sets of generators for the K-theory (by nontrivial self-adjoint idempotents and unitaries) and the K-homology (by nontrivial Fredholm modules) of the spheres $S_{q}^{N-1}$. We also construct the corresponding Chern characters in cyclic homology and cohomology and compute the pairing of K-theory with K-homology. On odd spheres (i. e., for $\mathrm{N}$ even) we exhibit unbounded Fredholm modules by means of a natural unbounded operator $D$ which, while failing to have compact resolvent, has bounded commutators with all elements in the algebra $A\left(S_{q}^{N-1}\right)$.
\end{abstract}




\section{Introduction}

There exists a growing literature devoted to the study of examples of "quantum" and "noncommutative" spaces. In this paper we shall dissect one class of these, the so called quantum Euclidean spheres $S_{q}^{N-1}$. They were first introduced in [9] as homogeneous spaces of quantum orthogonal groups $\mathrm{SO}_{\mathrm{q}}(\mathrm{N})$, which are R-matrix deformations of the usual orthogonal groups $\mathrm{SO}(\mathrm{N})$.

We shall regard the spheres $S_{\mathrm{q}}^{\mathrm{N}-1}$ as "noncommutative real affine varieties". For such an object, $X$, the algebra $A(X)$ is a finitely presented $*$-algebra. In contrast with classical algebraic geometry, there does not in general exist a topological point set $X$. Nevertheless, we regard $X$ as a noncommutative space and $A(X)$ as the algebra of polynomial functions on $X$.

In the classical case, one can consider the algebra of continuous functions on the underlying topological space of an affine variety. If $X$ is bounded, then this is a $C^{*}$-algebra and is the completion of $A(X)$. In general, one defines $\mathcal{C}(X)$ to be the $C^{*}$-algebraic completion of the $*$-algebra $A(X)$. To construct this, one considers all possible $*$-representations of $A(X)$ on a countably infinite-dimensional Hilbert space. Then the norm on $A(X)$ is defined as the supremum of the norms in all these representations and the $C^{*}$-algebra $\mathcal{C}(X)$ is the completion of $A(X)$ with respect to this norm. The $C^{*}$-algebra $\mathcal{C}(X)$ has the universal property that any *-homomorphism from $A(X)$ to a separable $C^{*}$-algebra factors through $\mathcal{C}(X)$. In particular, any $*$-representation of $A(X)$ extends to a representation of $\mathcal{C}(X)$.

For the noncommutative spaces at hand, the algebra $A\left(S_{q}^{N-1}\right)$ was described in [12] by means of a suitable self-adjoint idempotent (a matrix of functions whose square is itself). In the present paper we improve on this by giving a clearer and nicer presentation of $A\left(S_{q}^{N-1}\right)$ in terms of a self-adjoint unipotent (a matrix of functions whose square is the identity) which is defined recursively. We then exhibit all representations of the algebra $A\left(S_{q}^{N-1}\right)$ which in turn extend to the $C^{*}$-algebra $\mathrm{C}\left(\mathrm{S}_{\mathrm{q}}^{\mathrm{N}-1}\right)$.

The core of the paper is the study of generators of the K-homology and K-theory of the spheres $S_{q}^{N-1}$. The K-theory classes will be given by means of self-adjoint idempotents (naturally associated with the aforementioned unipotents) and of unitaries in algebras of matrices over $A\left(S_{q}^{N-1}\right)$. The K-homology classes will be given as (homotopy classes of) suitable 1-summable Fredholm modules.

For odd spheres (i.e. for $\mathrm{N}$ even) the odd $\mathrm{K}$-homology generators are first given in terms of unbounded Fredholm modules. These are given by means of a natural unbounded operator $\mathrm{D}$ which, while failing to have compact resolvent, has bounded commutators with all elements in the algebra $A\left(S_{q}^{2 n+1}\right)$.

On the way to computing the pairing of K-theory with K-homology, we will exhibit the Chern characters of the generators of the K-theory and K-homology, as elements in the cyclic homology $\mathrm{HC}_{*}\left[\mathrm{~A}\left(\mathrm{~S}_{\mathrm{q}}^{\mathrm{N}-1}\right)\right]$ and cyclic cohomology $\mathrm{HC}^{*}\left[\mathrm{~A}\left(\mathrm{~S}_{\mathrm{q}}^{\mathrm{N}-1}\right)\right]$ respectively.

Needless to say, the pairing is integral (it comes from a noncommutative index 
theorem). Furthermore, the non-vanishing of the pairing will testify to the nontriviality of the elements that we construct in both K-homology K-theory.

\section{Quantum Euclidean Spheres}

As we have already mentioned, the quantum Euclidean spheres, $S_{q}^{N-1}$, were introduced in [9] as quantum homogeneous spaces of the quantum orthogonal groups, $\mathrm{SO}_{\mathrm{q}}(\mathrm{N})$, the latter being R-matrix deformations of usual orthogonal groups $\mathrm{SO}(\mathrm{N})$.

We shall briefly recall that construction [15, 1, 12]. Let us start with the quantum Euclidean space $\mathbb{R}_{\mathrm{q}}^{\mathrm{N}}$. The $*$-algebra $A\left(\mathbb{R}_{\mathrm{q}}^{\mathrm{N}}\right)$ of its polynomial "functions" is generated by elements $\left\{\widetilde{x}_{0}=\widetilde{x}_{0}^{*}, x_{i}, x_{i}^{*}, i=1, \ldots, n\right\}$ for $N=2 n+1$ while for $N=2 n$ there is no $\widetilde{x}_{0}$ (the reason for the notation $\widetilde{x}_{0}$ will be clear shortly). These generators fulfill commutation relations,

$$
\begin{aligned}
x_{i} x_{j} & =q x_{j} x_{i}, \quad 0 \leq i<j \leq n, \\
x_{i}^{*} x_{j} & =q x_{j} x_{i}^{*}, \quad i \neq j, \\
{\left[x_{i}, x_{i}^{*}\right] } & = \begin{cases}{\left[\left(1-q^{-2}\right) /\left(1+q^{-2 \rho_{i-1}}\right)\right] r_{i-1}^{2}} & i>1, \\
0 & i=1, N=2 n, \\
\left(1-q^{-1}\right) \widetilde{x}_{0}^{2} & i=1, N=2 n+1,\end{cases}
\end{aligned}
$$

with $\rho_{i}=\frac{1}{2}-i$ or $\rho_{i}=1-i, i=1, \ldots, n$, according to whether $N$ is odd or even respectively. The elements $r_{i}^{2}, i=1, \ldots, n$, are given by,

$$
r_{i}^{2}=q^{-2 \rho_{i}} \chi^{i}\left(x^{i}\right)^{*}+q^{-2 \rho_{i-1}} \chi^{i-1}\left(\chi^{i-1}\right)^{*} \cdots+\left(\chi^{i-1}\right)^{*} \chi^{i-1}+\left(\chi^{i}\right)^{*} \chi^{i},
$$

and one can prove that $r^{2} \equiv r_{n}^{2}$ is central.

In the classical $(q=1)$ case, these $r_{i}^{2}$ 's are simply sums of squares of coordinates and fixing the value of $r^{2}$ corresponds to the definition of a sphere as the set of points at a fixed distance from the origin.

In our case, by fixing the value of $r^{2}$ we get the quantum Euclidean sphere $S_{q}^{N-1}$ of the corresponding "radius". Thus, the quantum Euclidean sphere $S_{q}^{\mathrm{N}-1}$ is naturally considered as a quantum subspace of the quantum Euclidean space $\mathbb{R}_{q}^{N}$ and the algebra $A\left(S_{q}^{N-1}\right)$ of polynomial functions on it is a quotient of the algebra $A\left(\mathbb{R}_{q}^{N}\right)$ by the ideal generated by the relation that fixes the radius. Furthermore, the natural coaction of $\mathrm{SO}_{\mathrm{q}}(\mathrm{N})$ on $\mathbb{R}_{\mathrm{q}}^{\mathrm{N}}$,

$$
\delta: A\left(\mathbb{R}_{\mathrm{q}}^{\mathrm{N}}\right) \rightarrow A\left(\mathrm{SO}_{\mathrm{q}}(\mathrm{N})\right) \otimes A\left(\mathbb{R}_{\mathrm{q}}^{\mathrm{N}}\right),
$$

preserves the radius $r^{2}, \delta\left(r^{2}\right)=1 \otimes r^{2}$, and yields a coaction of $\mathrm{SO}_{\mathrm{q}}(\mathrm{N})$ on $\mathrm{S}_{\mathrm{q}}^{\mathrm{N}-1}$.

We can simplify the relations (2.1) by rescaling one generator, $x_{0}:=\left(1+q^{-1}\right)^{-1 / 2} \widetilde{x}_{0}$. It is also simpler to use rescaled "partial radii" which are related to the $r_{i}^{2 \prime} s$ by,

$$
r_{i}^{2}=\left(1+q^{-2 \rho_{i}}\right) s_{i},
$$


and are given recursively by

$$
\begin{aligned}
& s_{i}:=s_{i-1}+x_{i}^{*} x_{i}=q^{-2} s_{i-1}+x_{i} x_{i}^{*}, \\
& s_{0}:=x_{0}^{2} .
\end{aligned}
$$

By using these new elements $s_{i}$ we can write the commutation relations of the generators $\left\{x_{0}=x_{0}^{*}, x_{i}, x_{i}^{*}, i=1, \ldots, n\right\}$ of the algebra $A\left(\mathbb{R}_{q}^{N}\right)$ as,

$$
\begin{aligned}
x_{i} x_{j} & =q x_{j} x_{i}, \quad 0 \leq i<j \leq n, \\
x_{i}^{*} x_{j} & =q x_{j} x_{i}^{*}, \quad i \neq j, \\
{\left[x_{i}, x_{i}^{*}\right] } & =\left(1-q^{-2}\right) s_{i-1},
\end{aligned}
$$

with the understanding that $x_{0}=0$ if $N=2 n$. We see that the equality of the two formulæ for $s_{i}$ in (2.4) is equivalent to the commutation relation (2.5b).

Given $N$, the algebras $A\left(S_{q}^{N-1}\right)$ for quantum spheres of different radii are isomorphic. We are thus free to normalize the radius to the most convenient choice. This is $s_{n}=1$ or equivalently $r^{2}=1+q^{2 n-1}$ or $r^{2}=1+q^{2 n-2}$ according to whether $N$ is odd (even spheres) or $\mathrm{N}$ is even (odd spheres).

The elements $s_{i} \in A\left(S_{q}^{2 n}\right)$ are self-adjoint and related as

$$
0 \leq s_{0} \leq \cdots \leq s_{n-1} \leq s_{n}=1 .
$$

From the commutation relations (2.5a) it follows for $i<j$ that $x_{i}^{*} x_{i} x_{j}=q^{2} x_{j} x_{i}^{*} x_{i}$; on the other hand $x_{j}^{*} x_{j} x_{i}=x_{i} x_{j}^{*} x_{j}$. With a little induction, we deduce that

$$
s_{i} x_{j}=\left\{\begin{array}{ll}
q^{2} x_{j} s_{i} & : i<j \\
x_{j} s_{i} & : i \geq j,
\end{array} \quad s_{i} x_{j}^{*}= \begin{cases}q^{-2} x_{j}^{*} s_{i} & : i<j \\
x_{j}^{*} s_{i} & : i \geq j\end{cases}\right.
$$

and that the $s_{i}$ 's are mutually commuting.

Looking at relations (2.5), we see that odd quantum Euclidean spheres $S_{q}^{2 n-1}$ are the same as the odd quantum spheres introduced in [19] (see also [20]) as noncommutative homogeneous spaces of quantum unitary groups $\mathrm{SU}_{\mathrm{q}}(\mathrm{n})$.

To our knowledge this simple fact, which was observed during a conversation with F. Bonechi and L. Dabrowski, has not been presented before. It extends the classical result that odd dimensional spheres are simultaneously homogeneous spaces of orthogonal and of unitary groups.

The presentation of the algebra $A\left(\mathbb{R}_{q}^{N}\right)$ in [9] involved the square root of the deformation parameter $\mathrm{q}$; this must therefore be positive in that construction. In our presentation no square roots are involved and we may take any value of $q \in \mathbb{R}$; however, we shall soon see that we may restrict the values of $q$ without loss of generality, due to the occurrence of natural isomorphisms.

In [12] it was shown that the defining relations of the algebra $A\left(S_{q}^{N-1}\right)$ of polynomial functions on $S_{q}^{N-1}$ are equivalent to the condition that a certain matrix over 
$A\left(S_{q}^{N-1}\right)$ be idempotent. This is also equivalent to the condition that another matrix be unipotent, as we shall explain presently.

First consider the even spheres $S_{q}^{2 n}$ for any integer $n>0$. The algebra $A\left(S_{q}^{2 n}\right)$ is generated by elements $\left\{x_{0}, x_{i}, x_{i}^{*}, i=1, \ldots, n\right\}$.

We recursively define self-adjoint matrices $\mathfrak{u}_{(2 n)} \in \operatorname{Mat}_{2^{n}}\left(\mathbb{C}\left\langle x_{0}, x_{i}, x_{i}^{*}, i=1, \ldots, n\right\rangle\right)$ for all $n$ by,

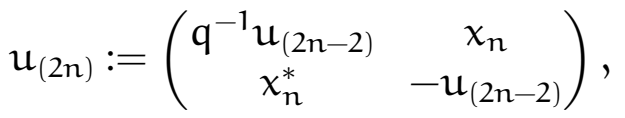

with $\mathfrak{u}_{(0)}=x_{0}$. The $*$-algebra $A\left(S_{q}^{2 n}\right)$ is then defined by the relations that $\mathfrak{u}_{(2 n)}$ is unipotent, $u_{(2 n)}^{2}=1$, and self-adjoint, $u_{(2 n)}^{*}=u_{(2 n)}$. That is, it is the quotient of the free $*$-algebra on $2 \mathrm{n}+1$ generators by these relations.

The self-adjointness relations merely give that $x_{i}^{*}$ is the adjoint of $x_{i}$ and $x_{0}$ is selfadjoint. Unipotency gives a matrix of $2^{2 n}$ relations, although many of these are vacuous or redundant. These can be deduced inductively from (2.7) which gives,

$$
\mathfrak{u}_{(2 \mathfrak{n})}^{2}=\left(\begin{array}{cc}
q^{-2} u_{(2 n-2)}^{2}+x_{n} x_{n}^{*} & q^{-1} u_{(2 n-2)} x_{n}-x_{n} u_{(2 n-2)} \\
q^{-1} x_{n}^{*} u_{(2 n-2)}-u_{(2 n-2)} x_{n}^{*} & u_{(2 n-2)}^{2}+x_{n}^{*} x_{n}
\end{array}\right) .
$$

The condition that $\mathfrak{u}_{(2 n)}^{2}=1$ means in particular that $\mathfrak{u}_{(2 n)}^{2}$ is diagonal with all the diagonal entries equal. Looking at (2.8), we see that the same must be true of $u_{2 n-2}^{2} \in \operatorname{Mat}_{2^{n-1}}\left(A\left(S_{q}^{2 n}\right)\right)$, and so on. Thus, the diagonal relations require that all the diagonal entries of (each) $\mathfrak{u}_{(2 j)}^{2}$ are equal. If this is true for $\mathfrak{u}_{(2 j-2)}^{2}$, then the relation for $\mathfrak{u}_{(2 j)}^{2}$ is that the same element (the diagonal entry) can be written in two different ways. This element is simply $s_{j}$ and the two ways of writing it are those given in (2.4).

Finally, $u_{(2 n)}^{2}=1$ gives the relation $s_{n}=1$.

The off-diagonal relations are $q^{-1} u_{(2 j-2)} x_{j}=x_{j} u_{(2 j-2)}$ and $q^{-1} x_{j}^{*} u_{(2 j-2)}=u_{(2 j-2)} x_{j}^{*}$ for every $j=1, \ldots, n$. Because the matrix $\mathfrak{u}_{(2 j-2)}$ is constructed linearly from all of the generators $x_{i}$ and $x_{i}^{*}$ for $i<j$, this conditions are equivalent to the commutation relations (2.5a).

In summary, up we see that the defining relations are all obtained from the unipotency condition, $\mathrm{u}_{(2 \mathrm{n})}^{2}=1$.

This presentation of the relations by the unipotency of $\mathfrak{u}_{(2 n)}$ is the easiest way to see that there is an isomorphism $A\left(S_{1 / q}^{2 n}\right) \cong A\left(S_{q}^{2 n}\right)$. The substitutions $q \leftrightarrow q^{-1}$, $x_{0} \rightarrow(-q)^{n} x_{0}$, and $x_{i} \rightarrow(-q)^{n-i} x_{i}^{*}$ are equivalent to conjugating $u_{(2 n)}$ by the antidiagonal matrix

$$
\left(\begin{array}{lllll} 
& & & & 1 \\
& & & 1 & \\
& & 1 & & \\
& & & & \\
1 & & &
\end{array}\right)
$$

and the result is unipotent and self-adjoint if and only if $\mathfrak{u}_{(2 n)}$ is; thus there is an isomorphism, $A\left(S_{1 / q}^{2 n}\right) \cong A\left(S_{q}^{2 n}\right)$. Because of this, we can assume that $|q|>1$ without loss of generality. 
Now consider the odd spheres $S_{q}^{2 n-1}$ for any integer $n>0$. We can construct a unipotent $\mathfrak{u}_{(2 n-1)} \in \operatorname{Mat}_{2^{n}}\left[A\left(S_{q}^{2 n-1}\right)\right]$, simply by setting $x_{0}=0$ in $\mathfrak{u}_{(2 n)}$. Once again, the unipotency condition, $\mathfrak{u}_{(2 n-1)}^{2}=1$, is equivalent to the relations defining the algebra $A\left(S_{q}^{2 n-1}\right)$ of polynomial functions on $S_{q}^{2 n-1}$. Again, one defines self-adjoint elements $s_{i} \in A\left(S_{q}^{2 n-1}\right)$ such that $s_{i}=s_{i-1}+x_{i}^{*} x_{i}=q^{-2} s_{i-1}+x_{i} x_{i}^{*}$ with now $s_{0}=x_{0}^{2}=0$. The commutation relations are again given by (2.5) but now (2.5b) gives in particular that the generator $x_{1}$ is normal,

$$
x_{1} x_{1}^{*}=x_{1}^{*} x_{1} \quad \text { in } \quad A\left(S_{q}^{2 n-1}\right) .
$$

The previous argument also shows that $A\left(S_{q}^{2 n-1}\right)$ is the quotient of $A\left(S_{q}^{2 n}\right)$ by the ideal generated by $x_{0}$; geometrically, this means that $S_{q}^{2 n-1}$ is a noncommutative subspace of $S_{q}^{2 n}$.

Because of the isomorphism $A\left(S_{1 / q}^{2 n}\right) \cong A\left(S_{q}^{2 n}\right)$, we have another isomorphism $A\left(S_{1 / q}^{2 n-1}\right) \cong A\left(S_{q}^{2 n-1}\right)$, and again we can assume that $|q|>1$ without any loss of generality.

\subsection{Interrelations}

The even sphere algebras each have an involutive automorphism

$$
\begin{gathered}
\sigma: A\left(S_{q}^{2 \mathfrak{n}}\right) \rightarrow A\left(S_{q}^{2 \mathfrak{n}}\right) \\
x_{0} \mapsto-x_{0}, \\
x_{j} \mapsto x_{j}, \quad j \neq 0 .
\end{gathered}
$$

Obviously, this corresponds to flipping (reflecting) the classical $S^{2 n}$ across the hyperplane $x_{0}=0$. The coinvariant algebra of $\sigma$ is the quotient of $A\left(S_{q}^{2 n}\right)$ by the ideal generated by $x_{0}$, which, as we have noted, is simply $A\left(S_{q}^{2 n-1}\right)$. Geometrically this means that $S_{q}^{2 n-1}$ is the "equator" of $S_{q}^{2 n}$ - the subspace fixed by the flip.

As for odd spheres, they have an action $\rho: \mathbb{T} \rightarrow \operatorname{Aut}\left[A\left(S^{2 n}\right)\right]$ of the torus group $\mathbb{T}$, defined by multiplying $x_{1}$ by a phase and leaving the other generators alone,

$$
\begin{aligned}
\rho(\lambda): & A\left(S_{q}^{2 n+1}\right) \rightarrow A\left(S_{q}^{2 n+1}\right) \\
& x_{1} \mapsto \lambda x_{1}, \\
& x_{j} \mapsto x_{j}, \quad j \neq 1 .
\end{aligned}
$$

The coinvariant algebra is given by setting $x_{1}=0$. Now, let $\mathfrak{u}_{(2 n+1)}^{\prime}$ be the matrix obtained by setting $x_{1}=0$ and relabeling $x_{2}$ as $x_{1}$, et cetera, in the matrix $u_{(2 n+1)}$. Then, $\mathfrak{u}_{(2 n+1)}^{\prime}$ is equivalent to tensoring $\mathfrak{u}_{(2 n-1)}$ with $\left(\begin{array}{ll}1 & 0 \\ 0 & 1\end{array}\right)$,

$$
\mathfrak{u}_{(2 n+1)}^{\prime}=u_{(2 n-1)} \otimes\left(\begin{array}{ll}
1 & 0 \\
0 & 1
\end{array}\right)
$$

and the result is unipotent if and only if $\mathfrak{u}_{(2 n-1)}$ is; that is the unipotency of $\mathfrak{u}_{(2 n+1)}^{\prime}$ yields all and only the same relations coming from the unipotency of $\mathfrak{u}_{(2 n-1)}$. This 
shows that $A\left(S_{q}^{2 n-1}\right)$ is the quotient of $A\left(S_{q}^{2 n+1}\right)$ by the $*$-ideal generated by $x_{1}$ and $S_{q}^{2 n-1}$ is the noncommutative subspace of $S_{q}^{2 n+1}$ fixed by the $\mathbb{T}$-action in (2.11).

There is also a way of realizing even spheres as noncommutative subspaces of odd ones. Consider $S_{q}^{2 n+1}$, set $x_{1}=x_{1}^{*}=x_{0}$ and relabel $x_{2}$ as $x_{1}$, et cetera; let $u_{(2 n+1)}^{\prime \prime}$ be the matrix obtained from $u_{(2 n+1)}$ with these substitutions. The matrix $u_{(2 n+1)}^{\prime \prime}$ is the same as $\mathfrak{u}_{(2 n)}$ in which we substitute

$$
x_{0} \rightarrow\left(\begin{array}{cc}
0 & x_{0} \\
x_{0} & 0
\end{array}\right), \quad x_{j} \rightarrow\left(\begin{array}{cc}
x_{j+1} & 0 \\
0 & x_{j+1}
\end{array}\right), \quad j \neq 0 .
$$

Then the unipotency of $u_{(2 n+1)}^{\prime \prime}$ yields precisely the same relations coming from the unipotency of $u_{(2 n)}$; this shows that $A\left(S_{q}^{2 n}\right)$ is the quotient of $A\left(S_{q}^{2 n+1}\right)$ by the $*$-ideal generated by $x_{1}-x_{1}^{*}$.

Thus, every sphere contains a smaller sphere of dimension one less; by following this tower of inclusions to its base, we see that every sphere contains a classical $S^{1}$, because the circle does not deform.

\section{Structure and Representations}

For each dimension $N$, we have a one parameter family of algebras $A\left(S_{q}^{N-1}\right)$ which, at $q=1$, gives $A\left(S_{1}^{N-1}\right)=A\left(S^{N-1}\right)$, the algebra of polynomial functions on a classical sphere $S^{N-1}$. It is possible to identify this one-parameter family of algebras to a fixed vector space and view the product as varying with the parameter. We can then construct a Poisson bracket on $A\left(S^{N-1}\right)$ from the first derivative of the product at the "classical" parameter value, $q=1$. The standard properties of a Poisson bracket (Leibniz and Jacobi identities) are simple consequences of associativity. The Poisson bracket is given geometrically by a Poisson bivector $\pi$, an antisymmetric contravariant 2-tensor. This in turn determines a symplectic foliation by the directions in which $\pi$ is nondegenerate.

In general, given such a one-parameter deformation from a commutative manifold $\mathcal{M}$ into noncommutative algebras, we can construct a Poisson bracket on functions. This Poisson algebra, $A(\mathcal{M})$ with the commutative product and the Poisson bracket, describes the deformation to first order. A deformation is essentially a path through an enormous space of possible algebras, and the Poisson algebra is just a tangent. Nevertheless, if the deformation is well behaved the Poisson algebra does indicate where it is heading. Here are some things that one can expect.

If $\pi$ vanishes along some subspace $X \subset \mathcal{M}$, then $\pi$ induces a trivial Poisson structure on $X$; i. e., $X$ is undeformed to first order. This suggests that it may be undeformed altogether. If so, then $X$ will be a classical subspace; that is, there will be a surjective homomorphism of the deformed algebra to the (undeformed) algebra of functions on $X$.

More generally, if $X \subset \mathcal{M}$ is a submanifold such that the restriction of $\pi$ to $X$ is tangent to $X$, then the restriction of functions to $X$ is a Poisson homomorphism, 
$A(\mathcal{M}) \rightarrow A(X)$; i. e., the deformation respects $X$ to first order. In this case it may be that some deformation of $\mathrm{X}$ is a "noncommutative subspace" of the deformation of $\mathcal{M}$. Algebraically speaking, this means that the algebra corresponding to $\mathrm{X}$ is a quotient of that corresponding to $\mathcal{M}$. Equivalently, the subalgebra of functions on $\mathcal{M}$ vanishing along $X$ correspond to an ideal in the deformation.

Suppose that the symplectic leaves of $\mathcal{M}$ are compact and $Y$ is the leaf space. Functions which are constant along the symplectic leaves can be identified with functions on $Y$. In this way, $A(Y)$ is the center of the Poisson algebra. That is, if $f \in A(Y)$ and $g \in A(\mathcal{M})$ then $\{f, g\}=0$. This suggests that the subalgebra $A(Y) \subset A(\mathcal{M})$ will be undeformed and will be the center of the deformed algebra.

More generally, if the symplectic foliation has a Hausdorff leaf space, $Y$, then $A(Y)$ acts by central multipliers on the Poisson algebra. That is for $f$ and $g$ functions on $\mathcal{M}$ and $h$ a function on $Y$, one has $\{h f, g\}=h\{f, g\}$. This suggests that $A(Y)$ my undeformed and will act by central multipliers on the deformation of $A(\mathcal{M})$. If so, then the deformed algebra will be the algebra of sections of a bundle of algebras over $\mathrm{Y}$. The fibers will be deformations of the symplectic leaves.

With these ideas in mind, consider some of the properties of the deformed spheres $S_{\mathrm{q}}^{\mathrm{N}-1}$.

We have seen that the $S_{q}^{2 n-1}$ noncommutative subspace of $S_{q}^{2 n}$ corresponds to the equator, the $S^{2 n-1} \subset S^{2 n}$ where $x_{0}=0$ and the Poisson bivector on $S^{2 n}$ is degenerate. On the remaining $S^{2 n} \backslash S^{2 n-1}$, the Poisson bivector is nondegenerate. So, topologically, we have a union of two copies of symplectic $\mathbb{R}^{2 n}$.

The manifold $\mathbb{R}^{2 n}$ has a unique symplectic structure, modulo isomorphism. This symplectic structure corresponds to an essentially unique deformation. If we complete to a $C^{*}$-algebra, then the deformation of $\mathcal{C}_{0}\left(\mathbb{R}^{2 \mathfrak{n}}\right)$ will be the algebra, $\mathcal{K}$, of compact operators on a countably infinite-dimensional Hilbert space.

Then, the kernel of the quotient map $A\left(S_{q}^{2 \mathfrak{n}}\right) \rightarrow A\left(S_{q}^{2 \mathfrak{n}-1}\right)$ should be a deformation of the subalgebra of functions on $S^{2 n}$ which vanish at the equator. If we complete to $C^{*}$-algebras, this should give us the direct sum of two copies of $\mathcal{K}$, one for each hemisphere. Thus we expect that the $C^{*}$-algebra $\mathcal{C}\left(S_{q}^{2 n}\right)$ will be an extension:

$$
0 \rightarrow \mathcal{K} \oplus \mathcal{K} \rightarrow \mathcal{C}\left(S_{\mathbf{q}}^{2 \mathfrak{n}}\right) \rightarrow \mathcal{C}\left(S_{\mathbf{q}}^{2 \mathfrak{n}-1}\right) \rightarrow 0 .
$$

In odd dimensions, the Poisson bivector is necessarily degenerate. However, the $S_{q}^{2 n-1}$ noncommutative subspace of $S_{q}^{2 n+1}$ corresponds classically to the Poisson bivector being more degenerate on $S^{2 n-1} \subset S^{2 n+1}$. It is of rank $2 n$ at most points, but of rank $2 n-2$ (or less) along $S^{2 n-1}$. The complement $S^{2 n+1} \backslash S^{2 n-1}$ has a symplectic foliation by $2 n$ dimensional leaves which is invariant under the $\mathbb{T}$ action; the simplest possibility is that this corresponds to the product in the identification

$$
S^{2 n+1} \backslash S^{2 n-1} \cong S^{1} \times \mathbb{R}^{2 n} .
$$

If we complete to $C^{*}$-algebras, then the deformation of this should be $\mathcal{C}\left(S^{1}\right) \otimes \mathcal{K}$. The kernel of the quotient map $A\left(S_{q}^{2 n+1}\right) \rightarrow A\left(S_{q}^{2 n-1}\right)$ should be this deformation, 
so we expect another extension,

$$
0 \rightarrow \mathcal{C}\left(S^{1}\right) \otimes \mathcal{K} \rightarrow \mathcal{C}\left(S_{q}^{2 n+1}\right) \rightarrow \mathcal{C}\left(S_{q}^{2 n-1}\right) \rightarrow 0 .
$$

These expectations are true. As we have mentioned, the odd dimensional spheres we are considering are equivalent to the "unitary" odd quantum spheres of Vaksman and Soibelman [19]. In [11] Hong and Szymański obtained the $C^{*}$-algebras $\mathcal{C}\left(S_{q}^{2 n+1}\right)$ as Cuntz-Krieger algebras of suitable graphs. From this construction they derived the extension (3.2). They also considered even spheres, defined as quotients of odd ones by the ideal generated by $x_{1}-x_{1}^{*}$. These are thus isomorphic to the even spheres we are considering here. They also obtained these as CuntzKrieger algebras and derived the extension (3.1). However, as explicitly stated in the introduction to [11], they were unable to realize even spheres as quantum homogeneous spaces of quantum orthogonal groups, thus also failing to realize that "unitary" and "orthogonal" odd quantum spheres are the same.

Representations of the odd dimensional spheres were constructed in [19]. The primitive spectra of all these spheres were compute in [11], which amounts to a classification of representations. The representations for quantum Euclidean spheres have also been constructed in [10] by thinking of them as quotient algebras of quantum Euclidean planes.

By using the properties we have just discussed, we shall present a clearer derivation of the representations. Indeed, the structure of the representations can be anticipated from the construction of $S_{q}^{N-1}$ via the extensions (3.1) and (3.2) and by remembering that an irreducible representation $\psi$ can be partially characterized by its kernel. Moreover, an irreducible representation of a $\mathrm{C}^{*}$-algebra restricts either to an irreducible or a trivial representation of any ideal; and conversely, an irreducible representation of an ideal extends to an irreducible representation of the $\mathrm{C}^{*}$-algebra (see for instance [8]).

For an even sphere $S_{q}^{2 n}$, the kernel of an irreducible representation $\psi$ will contain one or both of the copies of $\mathcal{K} \subset \mathcal{C}\left(S_{q}^{2 n}\right)$. If $\mathcal{K} \oplus \mathcal{K} \subseteq$ ker $\psi$, then $\psi$ factors through $\mathcal{C}\left(S_{q}^{2 n-1}\right)$ and is given by a representation of that algebra. If one copy of $\mathcal{K}$ is not in $\operatorname{ker} \psi$, then $\psi$ restricts to a representation of this $\mathcal{K}$. However, $\mathcal{K}$ has only one irreducible representation. Since $\mathcal{K}$ is an ideal in $\mathcal{C}\left(S_{q}^{2 \mathfrak{n}}\right)$, the unique irreducible representation of $\mathcal{K}$ uniquely extends to a representation of $\mathcal{C}\left(S_{q}^{2 n}\right)$ (with the other copy of $\mathcal{K}$ in its kernel).

Thus, we expect the irreducible representations of $S_{q}^{2 n}$ (up to isomorphism) to be:

1. all irreducible representations of $S_{q}^{2 n-1}$

2. a unique representation with kernel the second copy of $\mathcal{K}$

3. a unique representation with kernel the first copy of $\mathcal{K}$.

From the extension (3.1) we expect that the generator $x_{0}$ is a self-adjoint element of $\mathcal{K} \oplus \mathcal{K} \subset \mathcal{C}\left(S_{q}^{2 n}\right)$ and it should have almost discrete, real spectrum: it will therefore be a convenient tool for decomposing the Hilbert space in a representation.

Similarly, from the construction of $S_{q}^{2 n+1}$ by the extension (3.2), one can anticipate 
the structure of its representations. Firstly, if $\mathcal{C}\left(S^{1}\right) \otimes \mathcal{K} \subseteq \operatorname{ker} \psi$, then $\psi$ factors through $\mathrm{C}\left(S_{\mathrm{q}}^{2 \mathrm{n}-1}\right)$ and is really a representation of $S_{\mathrm{q}}^{2 \mathrm{n}-1}$.

Otherwise, $\psi$ restricts to an irreducible representation of $\mathcal{C}\left(S^{1}\right) \otimes \mathcal{K}$. This factorizes as the tensor product of an irreducible representation of $\mathcal{C}\left(\mathrm{S}^{1}\right)$ with one of $\mathcal{K}$. The irreducible representations of $\mathcal{C}\left(S^{1}\right)$ are simply given by the points of $S^{1}$, and as we have mentioned, $\mathcal{K}$ has a unique irreducible representation. The representations of $\mathcal{C}\left(S^{1}\right) \otimes \mathcal{K}$ are thus classified by the points of $S^{1}$. These representations extend uniquely from the ideal $\mathcal{C}\left(S^{1}\right) \otimes \mathcal{K}$ to the whole algebra $\mathcal{C}\left(S_{q}^{2 n+1}\right)$.

Thus, we expect the irreducible representations of $S_{q}^{2 n+1}$ (up to isomorphism) to be:

1. all irreducible representations of $S_{q}^{2 n-1}$

2. a family of representations parameterized by $S^{1}$.

In our construction of the representations, a simple identity regarding the spectra of operators will be especially useful (see, for instance [18]). If $x$ is an element of any $\mathrm{C}^{*}$-algebra, then

$$
\{0\} \cup \operatorname{Spec} x^{*} x=\{0\} \cup \operatorname{Spec} x x^{*} .
$$

\subsection{Even Sphere Representations}

To illustrate the general structure we shall start by describing the lowest dimensional case, namely $S_{\mathrm{q}}^{2}$. This is isomorphic to the so-called equator sphere of Podles̀ [16]. For this sphere, the representations were also constructed in [14] in a way close to the one presented here.

Let us then consider the sphere $S_{q}^{2}$.

As we have discussed, we expect that $x_{0}$ is a compact operator (in some faithful representation) and thus has an almost discrete, real spectrum. The relation $x_{0} x_{1}=$ $q x_{1} x_{0}$ suggests that $x_{1}$ and $x_{1}^{*}$ shift the eigenvalues of $x_{0}$. However, we cannot assume $a$ priori that $x_{0}$ has eigenvalues, let alone that eigenvectors form a complete basis of the Hilbert space. The relation $1=x_{0}^{2}+x_{1}^{*} x_{1}=q^{-2} x_{0}^{2}+x_{1} x_{1}^{*}$ shows that $x_{0}^{2} \leq 1$ and thus $\left\|x_{0}\right\| \leq 1$. As $x_{0}$ is self-adjoint, this shows that $\operatorname{Spec} x_{0} \subseteq[-1,1]$. By (3.3) we have also,

$$
\begin{aligned}
\{0\} \cup \operatorname{Spec} x_{1}^{*} x_{1} & =\{0\} \cup \operatorname{Spec} x_{1} x_{1}^{*} \\
\{0\} \cup \operatorname{Spec}\left(1-x_{0}^{2}\right) & =\{0\} \cup \operatorname{Spec}\left(1-q^{-2} x_{0}^{2}\right) \\
\{1\} \cup \operatorname{Spec} x_{0}^{2} & =\{1\} \cup q^{-2} \operatorname{Spec} x_{0}^{2} .
\end{aligned}
$$

Because we have assumed that $|q| \geq 1$, the only subsets of $[0,1]$ that satisfy this condition are $\{0\}$ and $\left\{0, q^{-2 k} \mid k=0,1, \ldots\right\}$.

If $x_{0} \neq 0 \in \mathcal{C}\left(S_{q}^{2}\right)$ then $\operatorname{Spec} x_{0}^{2}$ is the latter set. We cannot simply assume that $x_{0} \neq 0$, since not every $*$-algebra is a subalgebra of a $C^{*}$-algebra; however, our explicit representations will show that that is the case here.

Now let $\mathcal{H}$ be a separable Hilbert space and suppose that $\psi: A\left(S_{q}^{2}\right) \rightarrow \mathcal{L}(\mathcal{H})$ is an irreducible $*$-representation. 
If $\psi\left(x_{0}\right)=0$ then $1=\psi\left(x_{1}\right) \psi\left(x_{1}\right)^{*}=\psi\left(x_{1}\right)^{*} \psi\left(x_{1}\right)$. Thus $\psi\left(x_{1}\right)$ is unitary, and by the assumption of irreducibility, it is a number $\lambda \in \mathbb{C},|\lambda|=1$. So, $\mathcal{H}=\mathbb{C}$ and the representation is $\psi_{\lambda}^{(1)}$ defined by,

$$
\begin{aligned}
& \psi_{\lambda}^{(1)}\left(x_{0}\right)=0, \\
& \psi_{\lambda}^{(1)}\left(x_{1}\right)=\lambda, \quad \lambda \in S^{1} .
\end{aligned}
$$

Thus we have an $S^{1}$ worth of representations with $x_{0}$ in the kernel.

If $\psi\left(x_{0}\right) \neq 0$, then $1 \in \operatorname{Spec} x_{0}^{2}$; it is an isolated point in the spectrum and therefore an eigenvalue. For some sign \pm there exists a unit vector $|0\rangle \in \mathcal{H}$ such that $\psi\left(x_{0}\right)|0\rangle= \pm|0\rangle$. So, for $k=0,1, \ldots, \psi\left(x_{1}^{*}\right)^{k}|0\rangle$ is an eigenvector as well, because

$$
\psi\left(x_{0}\right) \psi\left(x_{1}^{*}\right)^{\mathrm{k}}|0\rangle=\mathrm{q}^{-\mathrm{k}} \psi\left(x_{1}^{* \mathrm{k}} x_{0}\right)|0\rangle= \pm \mathrm{q}^{-\mathrm{k}} \psi\left(x_{1}^{*}\right)^{\mathrm{k}}|0\rangle .
$$

By normalizing, we obtain a sequence of unit eigenvectors, recursively defined by

$$
|k\rangle:=\left(1-q^{-2 k}\right)^{-1 / 2} \psi\left(x_{1}^{*}\right)|k-1\rangle \text {. }
$$

We have thus two representations $\psi_{+}^{(2)}$ and $\psi_{-}^{(2)}$, and direct computation shows that

$$
\begin{aligned}
& \psi_{ \pm}^{(2)}\left(x_{0}\right)|k\rangle= \pm q^{-k}|k\rangle \\
& \psi_{ \pm}^{(2)}\left(x_{1}\right)|k\rangle=\left(1-q^{-2 k}\right)^{1 / 2}|k-1\rangle \\
& \psi_{ \pm}^{(2)}\left(x_{1}^{*}\right)|k\rangle=\left(1-q^{-2(k+1)}\right)^{1 / 2}|k+1\rangle .
\end{aligned}
$$

The eigenvectors $\{|k\rangle \mid k=0,1, \ldots\}$ are mutually orthogonal because they have distinct eigenvalues, and by the assumption of irreducibility form a basis for $\mathcal{H}$.

Notice that any power of $\psi_{ \pm}^{(2)}\left(x_{0}\right)$ is a trace class operator, while this is not the case for the operators $\psi_{ \pm}^{(2)}\left(x_{1}\right)$ and $\psi_{ \pm}^{(2)}\left(x_{1}^{*}\right)$ nor for any of their powers.

Note also that the representations (3.5) are related by the automorphism $\sigma$ in (2.10), as

$$
\psi_{ \pm}^{(2)} \circ \sigma=\psi_{\mp}^{(2)} \text {. }
$$

If we set a value of $q$ with $|q|<1$ in (3.5), the operators would be unbounded. This is the reason for assuming that $|q|>1$. The assumption was used in computing Spec $x_{0}$. Not only is $\left\|x_{0}\right\| \leq 1$, but by a similar calculation $\left\|x_{0}\right\| \leq|q|$. Which bound is more relevant obviously depends on whether $q$ is greater or less than 1 .

For $|q|<1$ the appropriate formulæ for the representations can be obtained from (3.5) by replacing the index $k$ with $-k-1$. As a consequence, the role of $x_{1}$ and $x_{1}^{*}$ as lowering and raising operators is exchanged.

Let us then turn to the general even spheres $S_{q}^{2 n}$.

The structure of the representations is similar to that for $S_{q}^{2}$, but more complicated. The element $x_{0}$ is no longer sufficient to completely decompose the Hilbert space of the representation and we need to use all the commuting self-adjoint elements $s_{i} \in A\left(S_{q}^{2 n}\right)$ defined in (2.4). 
Using the formulæ for $1=s_{n}=s_{n-1}+x_{n}^{*} x_{n}=q^{-2} s_{n-1}+x_{n} x_{n}^{*}$ and (3.3) we get,

$$
\begin{aligned}
\{0\} \cup \operatorname{Spec} x_{n}^{*} x_{n} & =\{0\} \cup \operatorname{Spec} x_{n} x_{n}^{*} \\
\{0\} \cup \operatorname{Spec}\left(1-s_{n-1}\right) & =\{0\} \cup \operatorname{Spec}\left(1-q^{-2} s_{n-1}\right) \\
\{1\} \cup \operatorname{Spec} s_{n-1} & =\{1\} \cup q^{-2} \operatorname{Spec} s_{n-1} .
\end{aligned}
$$

Therefore either $s_{n-1}=0 \in \mathcal{C}\left(S_{q}^{2 n}\right)$ or Spec $s_{n-1}=\left\{0, q^{-2 k} \mid k=0,1, \ldots\right\}$.

Now suppose that $\psi: \mathrm{A}\left(S_{\mathbf{q}}^{2 \mathfrak{n}}\right) \rightarrow \mathcal{L}(\mathcal{H})$ is an irreducible $*$-representation.

If $\psi\left(x_{0}\right)=0$, then $\psi$ factors through $A\left(S_{q}^{2 n-1}\right)$. Thus $\psi$ is an irreducible representation of $A\left(S_{\mathrm{q}}^{2 n-1}\right)$; these will be discussed later.

If $\psi\left(x_{0}\right) \neq 0$, then $\psi\left(s_{0}\right) \neq 0$, and by the relations (2.6), all of the $\psi\left(s_{i}\right)^{\prime}$ s are nonzero. So, Spec $\psi\left(s_{n-1}\right)=\left\{0, q^{-2 k} \mid k=0,1, \ldots\right\}$ and in particular, 1 is an eigenvalue of $\psi\left(s_{n-1}\right)$. Because $s_{n-1}$ commutes with all the generators except $x_{n}$ and $x_{n}^{*}$, these generators preserve the corresponding eigenspace. The same argument as for $s_{n-1}$ shows that the restriction of $\psi\left(s_{n-2}\right)$ to this eigenspace has the same spectrum; in particular, 1 is an eigenvalue. There is a simultaneous eigenspace of $\psi\left(s_{n-1}\right)$ and $\psi\left(s_{n-2}\right)$ with eigenvalue 1 for both. Proceeding in this way, we find that there is a simultaneous eigenspace with eigenvalue 1 for all the $\psi\left(s_{i}\right)^{\prime} s$. That is, there must exist a unit vector $|0, \ldots, 0\rangle \in \mathcal{H}$ such that $\psi\left(s_{i}\right)|0, \ldots, 0\rangle=|0, \ldots, 0\rangle$ for all $i$ and $\psi\left(x_{0}\right)|0, \ldots, 0\rangle= \pm|0, \ldots, 0\rangle$. We construct more unit vectors by letting $\left|k_{0}, \ldots, k_{n-1}\right\rangle$ be

$$
\psi\left(x_{1}^{*}\right)^{k_{0}} \ldots \psi\left(x_{n}^{*}\right)^{k_{n-1}}|0, \ldots, 0\rangle
$$

modulo a positive normalizing factor. Using the commutation relations between $s_{i}$ 's and $x_{j}^{* \prime} s$ we get that,

$$
\psi\left(s_{i}\right)\left|k_{0}, \ldots, k_{n-1}\right\rangle=q^{-2\left(k_{i}+\cdots+k_{n-1}\right)}\left|k_{0}, \ldots, k_{n-1}\right\rangle .
$$

In summary, $x_{i}$ lowers $k_{i-1}, x_{i}^{*}$ raises $k_{i-1}$, and $s_{i}$ measures the sum $k_{i}+\cdots+k_{n-1}$. The correct normalizing factors can be determined from,

$$
\begin{aligned}
\left\langle k_{0}, \ldots, k_{n-1}\left|\psi\left(x_{i} x_{i}^{*}\right)\right| k_{0}, \ldots, k_{n-1}\right\rangle & =\left\langle k_{0}, \ldots, k_{n-1}\left|\psi\left(s_{i}-s_{i-1}\right)\right| k_{0}, \ldots, k_{n-1}\right\rangle \\
& =\left(1-q^{-2 k_{i-1}}\right) q^{-2\left(k_{i}+\cdots+k_{n-1}\right)} .
\end{aligned}
$$

Thus we get two representations $\psi_{ \pm}^{(2 n)}$ defined by,

$\psi_{ \pm}^{(2 n)}\left(x_{0}\right)\left|k_{0}, \ldots, k_{n-1}\right\rangle= \pm q^{-\left(k_{0}+\cdots+k_{n-1}\right)}\left|k_{0}, \ldots, k_{n-1}\right\rangle$

$\psi_{ \pm}^{(2 n)}\left(x_{i}\right)\left|k_{0}, \ldots, k_{n-1}\right\rangle=\left(1-q^{-2 k_{i-1}}\right)^{1 / 2} q^{-\left(k_{i}+\cdots+k_{n-1}\right)}\left|k_{0}, \ldots, k_{i-1}-1, \ldots, k_{n-1}\right\rangle$

$\psi_{ \pm}^{(2 n)}\left(x_{i}^{*}\right)\left|k_{0}, \ldots, k_{n-1}\right\rangle=\left(1-q^{-2\left(k_{i-1}+1\right)}\right)^{1 / 2} q^{-\left(k_{i}+\cdots+k_{n-1}\right)}\left|k_{0}, \ldots, k_{i-1}+1, \ldots, k_{n-1}\right\rangle$

with $i=1, \ldots, n$. The assumption of irreducibility implies that the collection of vectors $\left\{\left|k_{0}, \ldots, k_{n-1}\right\rangle, k_{i} \geq 0\right\}$ constitute a complete basis for $\mathcal{H}$.

As before, the two representations (3.7) are related by the automorphism $\sigma$, as

$$
\psi_{ \pm}^{(2 n)} \circ \sigma=\psi_{\mp}^{(2 n)} .
$$


Again the formulæ (3.7) for the representations are for $|q|>1$; and again the representations for $|q|<1$ can be obtained by replacing all indices $k_{i}$ with $-k_{i}-1$.

In all of the irreducible representations of $A\left(S_{q}^{2 n}\right)$, the representative of $x_{0}$ is compact; in fact it is trace class. We can deduce from this that the $C^{*}$-ideal generated by $\psi_{ \pm}^{(2 \mathfrak{n})}\left(x_{0}\right)$ in $\mathcal{C}\left(S_{q}^{2 n}\right)$ is isomorphic to $\mathcal{K}(\mathcal{H})$, the ideal of all compact operators on $\mathcal{H}$. By using the continuous functional calculus, we can apply any function $f \in \mathcal{C}[-1,1]$ to $x_{0}$. If $f$ is supported on $[0,1]$, then $f\left(x_{0}\right) \in \operatorname{ker} \psi_{-}^{(2 n)}$. Likewise if $f$ is supported in $[-1,0]$, then $f\left(x_{0}\right) \in \operatorname{ker} \psi_{+}^{(2 n)}$. From this we deduce that the $C^{*}$-ideal generated by $x_{0}$ in $\mathcal{C}\left(S_{q}^{2 n}\right)$ is $\mathcal{K} \oplus \mathcal{K}$. One copy of $\mathcal{K}$ is $\operatorname{ker} \psi_{+}^{(2 n)}$; the other is $\operatorname{ker} \psi_{-}^{(2 n)}$. This shows that the extension (3.1) is correct.

\subsection{Odd Sphere Representations}

Again, to illustrate the general strategy we shall work out in detail the simplest case, that of the sphere $S_{\mathrm{q}}^{3}$. This can be identified with the underlying noncommutative space of the quantum group $\mathrm{SU}_{\mathrm{q}}(2)$ and as such the representations of the algebra are well known [21].

The generators $\left\{x_{i}, x_{i}^{*} \mid i=1,2\right\}$ of the algebra $A\left(S_{q}^{3}\right)$ satisfy the commutation relations $x_{1} x_{2}=q x_{2} x_{1}, x_{i}^{*} x_{j}=q x_{j} x_{i}^{*}, i \neq j,\left[x_{1}, x_{1}^{*}\right]=0$, and $\left[x_{2}, x_{2}^{*}\right]=\left(1-q^{-2}\right) x_{1} x_{1}^{*}$. Furthermore, there is the sphere relation $1=x_{2}^{*} x_{2}+x_{1}^{*} x_{1}=x_{2} x_{2}^{*}+q^{-2} x_{1} x_{1}^{*}$.

The normal generator $x_{1}$ plays much the same role for the representations of $S_{q}^{3}$ that $x_{0}$ does for those of $S_{q}^{2}$. The sphere relation shows that $\left\|x_{1}\right\| \leq 1$ and

$$
\begin{aligned}
\{0\} \cup \operatorname{Spec} x_{2}^{*} x_{2} & =\{0\} \cup \operatorname{Spec} x_{2} x_{2}^{*} \\
\{0\} \cup \operatorname{Spec}\left(1-x_{1}^{*} x_{1}\right) & =\{0\} \cup \operatorname{Spec}\left(1-q^{-2} x_{1} x_{1}^{*}\right) \\
\{1\} \cup \operatorname{Spec} x_{1}^{*} x_{1} & =\{1\} \cup q^{-2} \operatorname{Spec} x_{1}^{*} x_{1},
\end{aligned}
$$

which shows that either $x_{1}=0$ or $\operatorname{Spec} x_{1}^{*} x_{1}=\left\{0, q^{-2 k} \mid k=0,1, \ldots\right\}$.

Let $\psi: A\left(S_{q}^{3}\right) \rightarrow \mathcal{L}(\mathcal{H})$ be an irreducible $*$-representation.

If $\psi\left(x_{1}\right)=0$ then the relations reduce to $1=\psi\left(x_{2}\right) \psi\left(x_{2}\right)^{*}=\psi\left(x_{2}\right)^{*} \psi\left(x_{2}\right)$. Thus $\psi\left(x_{2}\right)$ is unitary and by the assumption of irreducibility, it is a scalar, $\psi\left(x_{2}\right)=\lambda \in \mathbb{C}$ with $|\lambda|=1$. Thus, as before, we have an $S^{1}$ of representations of this kind.

If $\psi\left(x_{1}\right) \neq 0$, then $1 \in \operatorname{Spec} \psi\left(x_{1}^{*} x_{1}\right)$ and is an isolated point in the spectrum. Thus, there exists a unit vector $|0\rangle \in \mathcal{H}$ such that $\psi\left(x_{1}^{*} x_{1}\right)|0\rangle=|0\rangle$, and by the assumption of irreducibility, there is some $\lambda \in \mathbb{C}$ with $|\lambda|=1$ such that $\psi\left(x_{1}\right)|0\rangle=\lambda|0\rangle$. We see then that $\psi\left(x_{2}^{*}\right)^{k}|0\rangle$ is an eigenvector

$$
\psi\left(x_{1}\right) \psi\left(x_{2}^{*}\right)^{k}|0\rangle=q^{-k} \psi\left(x_{2}^{* k} x_{1}\right)|0\rangle=\lambda q^{-k} \psi\left(x_{2}^{*}\right)^{k}|0\rangle .
$$

By normalizing, we get a sequence of unit eigenvectors recursively defined by

$$
|k\rangle:=\left(1-q^{-2 k}\right)^{-1 / 2} \psi\left(x_{2}^{*}\right)|k-1\rangle .
$$


A family of representations $\psi_{\lambda}^{(3)}, \lambda \in S^{1}$, is then defined by

$$
\begin{aligned}
\psi_{\lambda}^{(3)}\left(x_{1}\right)|k\rangle & =\lambda q^{-k}|k\rangle, \\
\psi_{\lambda}^{(3)}\left(x_{1}^{*}\right)|k\rangle & =\bar{\lambda} q^{-k}|k\rangle, \\
\psi_{\lambda}^{(3)}\left(x_{2}\right)|k\rangle & =\left(1-q^{-2 k}\right)^{1 / 2}|k-1\rangle, \\
\psi_{\lambda}^{(3)}\left(x_{2}^{*}\right)|k\rangle & =\left(1-q^{-2(k+1)}\right)^{1 / 2}|k+1\rangle .
\end{aligned}
$$

We notice that any power of $\psi_{\lambda}^{(3)}\left(x_{1}\right)$ or $\psi_{\lambda}^{(3)}\left(x_{1}^{*}\right)$ is a trace class operator, while this is not the case for the operators $\psi_{\lambda}^{(3)}\left(x_{2}\right)$ and $\psi_{\lambda}^{(3)}\left(x_{2}^{*}\right)$ nor for any of their powers.

Consider the general odd spheres $S_{q}^{2 n+1}$ and let $\psi: A\left(S_{q}^{2 n+1}\right) \rightarrow \mathcal{L}(\mathcal{H})$ be an irreducible representation.

If $\psi\left(x_{1}\right)=0$ then $\psi$ factors through $A\left(S_{q}^{2 n-1}\right)$ and is an irreducible representation of that algebra.

If $\psi\left(x_{1}\right) \neq 0$ then $\psi\left(s_{1}\right) \neq 0, \psi\left(s_{2}\right) \neq 0$, et cetera. By the same arguments as for $S_{q}^{2 n}$, there must exist a simultaneous eigenspace with eigenvalue 1 for all of $s_{1}, \ldots s_{n}$. By the assumption of irreducibility, this eigenspace is 1-dimensional. Let $|0, \ldots, 0\rangle \in \mathcal{H}$ be a unit vector in this eigenspace. Then $s_{i}|0, \ldots, 0\rangle=|0, \ldots, 0\rangle$ for $i=1, \ldots, n$. The restriction of $\psi\left(x_{1}\right)$ to this subspace is unitary and thus for some $\lambda \in \mathbb{C}$ with $|\lambda|=1, \psi\left(x_{1}\right)|0, \ldots, 0\rangle=\lambda|0, \ldots, 0\rangle$. We can construct more simultaneous eigenvectors of the $s_{i}{ }^{\prime}$ s. Define $\left|k_{1}, \ldots, k_{n}\right\rangle$ to be

$$
\psi\left(x_{2}\right)^{k_{1}} \ldots \psi\left(x_{n+1}\right)^{k_{n}}|0, \ldots, 0\rangle
$$

modulo a positive normalizing constant. Then

$$
\psi\left(x_{1}\right)\left|k_{1}, \ldots, k_{n}\right\rangle=\lambda\left|k_{1}, \ldots, k_{n}\right\rangle
$$

and

$$
\psi\left(s_{i}\right)\left|k_{1}, \ldots, k_{n}\right\rangle=q^{-2\left(k_{i}+\cdots+k_{n}\right)}\left|k_{1}, \ldots, k_{n}\right\rangle .
$$

Working out the normalization, this gives a family of representations $\psi_{\lambda}^{(2 n+1)}$ by,

$$
\begin{aligned}
& \psi_{\lambda}^{(2 n+1)}\left(x_{1}\right)\left|k_{1}, \ldots, k_{n}\right\rangle=\lambda q^{-\left(k_{1}+\cdots+k_{n}\right)}\left|k_{1}, \ldots, k_{n}\right\rangle \\
& \psi_{\lambda}^{(2 n+1)}\left(x_{1}^{*}\right)\left|k_{1}, \ldots, k_{n}\right\rangle=\bar{\lambda} q^{-\left(k_{1}+\cdots+k_{n}\right)}\left|k_{1}, \ldots, k_{n}\right\rangle \\
& \psi_{\lambda}^{(2 n+1)}\left(x_{i}\right)\left|k_{1}, \ldots, k_{n}\right\rangle=\left(1-q^{-2 k_{i-1}}\right)^{1 / 2} q^{-\left(k_{i}+\cdots+k_{n}\right)}\left|k_{1}, \ldots, k_{i-1}-1, \ldots, k_{n}\right\rangle, \\
& \psi_{\lambda}^{(2 n+1)}\left(x_{i}^{*}\right)\left|k_{1}, \ldots, k_{n}\right\rangle=\left(1-q^{-2\left(k_{i-1}+1\right)}\right)^{1 / 2} q^{-\left(k_{i}+\cdots+k_{n}\right)}\left|k_{1}, \ldots, k_{i-1}+1, \ldots, k_{n}\right\rangle,
\end{aligned}
$$

for $i=2, \ldots, n+1$. The assumption of irreducibility implies that the vectors $\left\{\left|k_{1}, \ldots, k_{n}\right\rangle, k_{i} \geq 0\right\}$ form an orthonormal basis of $\mathcal{H}$.

As for the even case, the formulæ (3.10) give bounded operators only for $|q|>1$; and as before, the representations for $|q|<1$ can be obtained by replacing all indices $k_{i}$ with $-k_{i}-1$. 
Again, as in the even case, we can verify that $\psi_{\lambda}^{(2 n+1)}\left(x_{1}\right)$ is compact (indeed, trace class) and that the ideal generated by $\psi_{\lambda}^{(2 n+1)}\left(x_{1}\right)$ in the $C^{*}$-algebra completion of the image $\psi_{\lambda}^{(2 n+1)}\left(A\left(S_{q}^{2 n+1}\right)\right)$ is $\mathcal{K}(\mathcal{H})$. The representations $\psi_{\lambda}^{(2 n+1)}$ can be assembled into a single representation by adjointable operators on a Hilbert $\mathcal{C}\left(S^{1}\right)$-module. With this we can verify that the ideal generated by $x_{1}$ in $\mathcal{C}\left(S_{q}^{2 n+1}\right)$ is $\mathcal{C}\left(S^{1}\right) \otimes \mathcal{K}$ and this fact verifies the extension (3.2).

Putting together the results for even and odd spheres, we get a complete picture of the set of irreducible representations of all these spheres.

For the odd spheres $S_{q}^{2 n+1}$, the set of irreducible representations (or equivalently, the primitive spectrum of $\left.\mathcal{C}\left(S_{q}^{2 n+1}\right)\right)$ is indexed by the union of $n+1$ copies of $S^{1}$. These run from the representations $\psi_{\lambda}^{(2 \mathfrak{n}+1)}$ of $S_{q}^{2 n+1}$ given in (3.10) down to the one dimensional representations $\psi_{\lambda}^{(1)}$ that factor through $\mathcal{C}\left(S^{1}\right)$.

For the even spheres $S_{q}^{2 n}$, the set of irreducible representations (or equivalently, the primitive spectrum of $\mathcal{C}\left(S_{q}^{2 n}\right)$ ) is indexed by the union of $n$ copies of $S^{1}$ and 2 points. The isolated points correspond to the 2 representations $\psi_{ \pm}^{(2 \mathfrak{n})}$ specific to $S_{q}^{2 n}$ and given in (3.7); the circles correspond to representations $\psi_{\lambda}^{(2 m+1)}$ coming from lower odd dimensional spheres, down to $S^{1}$.

\section{K-homology and K-theory}

We are now ready to study the K-homology and K-theory of the quantum Euclidean spheres $S_{q}^{N-1}$. The K-homology classes will be given by Fredholm modules using the representations constructed previously while the K-theory classes will be given by means of suitable idempotents and unitaries.

In fact, in order to compute the pairing of K-theory with K-homology, it is more convenient to first compute the Chern characters and then use the pairing between cyclic homology and cohomology [4]. Thus, together with the generators of K-theory and K-homology we shall also construct the associated Chern characters in the cyclic homology $\mathrm{HC}_{*}\left[\mathrm{~A}\left(\mathrm{~S}_{\mathbf{q}}^{\mathrm{N}-1}\right)\right]$ and cyclic cohomology $\mathrm{HC}^{*}\left[\mathrm{~A}\left(\mathrm{~S}_{\mathbf{q}}^{\mathrm{N}-1}\right)\right]$ respectively.

It is worth recalling the K-theory and homology of the classical spheres.

For an even dimensional sphere $S^{2 n}$, the groups are

$$
\begin{array}{ll}
K^{0}\left(S^{2 \mathfrak{n}}\right) \cong \mathbb{Z}^{2}, & \mathrm{~K}^{1}\left(S^{2 \mathfrak{n}}\right)=0, \\
\mathrm{~K}_{0}\left(S^{2 \mathfrak{n}}\right) \cong \mathbb{Z}^{2}, & \mathrm{~K}_{1}\left(S^{2 \mathfrak{n}}\right)=0 .
\end{array}
$$

One generator of the K-theory [1] $\in \mathrm{K}^{0}\left(\mathrm{~S}^{2 \mathfrak{n}}\right)$ is given by the trivial 1-dimensional bundle. The other generator of $\mathrm{K}^{0}\left(\mathrm{~S}^{2 \mathfrak{n}}\right)$ is the left handed spinor bundle. One $\mathrm{K}$-homology generator $[\varepsilon] \in \mathrm{K}_{0}\left(\mathrm{~S}^{2 \mathfrak{n}}\right)$ is "trivial" and is the push-forward of the generator of $K_{0}(*) \cong \mathbb{Z}$ by the inclusion $\iota: * \hookrightarrow S^{2 n}$ of a point (any point) into the sphere. The other generator, $[\mu] \in \mathrm{K}_{0}\left(S^{2 \mathrm{n}}\right)$, is the K-orientation of $S^{2 \mathrm{n}}$ given by its structure as a spin manifold [四]. 
For an odd dimensional sphere, the groups are

$$
\begin{array}{ll}
\mathrm{K}^{0}\left(S^{2 n+1}\right) \cong \mathbb{Z}, & \mathrm{K}^{1}\left(S^{2 n+1}\right) \cong \mathbb{Z}, \\
\mathrm{K}_{0}\left(S^{2 n+1}\right) \cong \mathbb{Z}, & \mathrm{K}_{1}\left(S^{2 n+1}\right) \cong \mathbb{Z} .
\end{array}
$$

The generator [1] $\in \mathrm{K}^{0}\left(S^{2 n+1}\right)$ is the trivial 1-dimensional bundle. The generator of $K^{1}\left(S^{2 n+1}\right)$ is a nontrivial unitary matrix-valued function on $S^{2 n+1}$. The generator $[\varepsilon] \in \mathrm{K}_{0}\left(S^{2 n+1}\right)$ is again the "trivial" element given by the inclusion of a point. The generator $[\mu] \in K_{1}\left(S^{2 \mathfrak{n}+1}\right)$ is the K-orientation of $S^{2 \mathfrak{n}+1}$ given by its structure as a spin manifold [4].

There is a natural pairing between K-homology and K-theory. If we pair $[\varepsilon]$ with a vector bundle we get the rank of the vector bundle, i. e. the dimension of its fibers. If we pair $[\mu]$ with a vector bundle it gives the "degree" of the bundle, a measure of its nontriviality. Similarly, pairing with $[\mu]$ measures the nontriviality of a unitary.

The K-theory and K-homology of the quantum Euclidean spheres are isomorphic to that of the classical spheres; that is, for any $\mathrm{N}$ and $\mathrm{q}, \mathrm{K}_{*}\left[\mathrm{C}\left(\mathrm{S}_{\mathrm{q}}^{\mathrm{N}-1}\right)\right] \cong \mathrm{K}^{*}\left(\mathrm{~S}^{\mathrm{N}-1}\right)$ and $\mathrm{K}^{*}\left[\mathcal{C}\left(\mathrm{S}_{\mathrm{q}}^{\mathrm{N}-1}\right)\right] \cong \mathrm{K}_{*}\left(\mathrm{~S}^{\mathrm{N}-1}\right)$.

In the case of K-theory, this was proven by Hong and Szymański in [11] using their construction of the $\mathrm{C}^{*}$-algebras as Cuntz-Krieger algebras of graphs. The groups $\mathrm{K}_{0}$ and $\mathrm{K}_{1}$ were given as the cokernel and the kernel respectively, of a matrix canonically associated with the graph. The result for K-homology can be proven using the same techniques $\left[\nabla,[7]\right.$ : the groups $\mathrm{K}^{0}$ and $\mathrm{K}^{1}$ are now given as the kernel and the cokernel respectively, of the transposed matrix. The K-theory and the K-homology for the particular case of $S_{q}^{2}$ (in fact for all Podles spheres $S_{q c}^{2}$ ) was worked out in [14]) while for $\mathrm{S}^{3} \cong \mathrm{SU}_{\mathrm{q}}(2)$ it was spelled out in [13].

\subsection{K-homology}

Because the K-homology of these deformed spheres is isomorphic to the K-homology of the ordinary spheres, we need to construct two independent generators. First consider the "trivial" generator of $\mathrm{K}^{0}\left[\mathrm{C}\left(\mathrm{S}_{\mathrm{q}}^{\mathrm{N}-1}\right)\right]$. This can be constructed in a manner closely analogous to the undeformed case.

As we have just described, the trivial generator of $\mathrm{K}_{0}\left(\mathrm{~S}^{\mathrm{N}-1}\right)$ is the image of the generator of the K-homology of a point by the functorial map $\mathrm{K}_{*}(\mathrm{\iota}): \mathrm{K}_{0}(*) \rightarrow \mathrm{K}_{0}\left(\mathrm{~S}^{\mathrm{N}-1}\right)$, where $\iota: * \hookrightarrow S^{N-1}$ is the inclusion of a point into the sphere. The quantum Euclidean spheres do not have as many points, but they do have some. We have seen that the relations among the various spheres always include a homomorphism $A\left(S_{q}^{N-1}\right) \rightarrow A\left(S^{1}\right)$. Equivalently, every $S_{q}^{N-1}$ has a circle $S^{1}$ as a classical subspace; thus for every $\lambda \in S^{1}$ there is a point, i. e., the homomorphism $\psi_{\lambda}^{(1)}: \mathcal{C}\left(S_{q}^{N-1}\right) \rightarrow \mathbb{C}$. We can construct an element $\left[\varepsilon_{\lambda}\right] \in \mathrm{K}^{0}\left[\mathcal{C}\left(S_{\mathrm{q}}^{\mathrm{N}-1}\right)\right]$ by pulling back the generator of $\mathrm{K}^{0}(\mathbb{C})$ by $\psi_{\lambda}^{(1)}$. This construction factors through $\mathrm{K}_{0}\left(S^{1}\right)$. Because $S^{1}$ is path connected, the points of $S^{1}$ all define homotopic (and hence K-homologous) Fredholm 
modules. Thus there is a single $\mathrm{K}$-homology class $\left[\varepsilon_{\lambda}\right] \in \mathrm{K}^{0}\left[\mathrm{C}\left(\mathrm{S}_{\mathbf{q}}^{\mathrm{N}-1}\right)\right]$, independent of $\lambda \in \mathrm{S}^{1}$.

The canonical generator of $\mathrm{K}^{0}(\mathbb{C})$ is given by the following Fredholm module: The Hilbert space is $\mathbb{C}$; the grading operator is $\gamma=1$; the representation is the obvious representation of $\mathbb{C}$ on $\mathbb{C}$; the Fredholm operator is 0 . If we pull this back to $\mathrm{K}^{0}\left[\mathcal{C}\left(S_{\mathrm{q}}^{\mathrm{N}-1}\right)\right]$ using $\psi_{\lambda}^{(1)}$, then the Fredholm module $\varepsilon_{\lambda}$ is given in the same way but with $\psi_{\lambda}^{(1)}$ for the representation.

Given this construction of $\varepsilon_{\lambda}$, it is straightforward to compute its Chern character $\operatorname{ch}^{*}\left(\varepsilon_{\lambda}\right) \in \mathrm{HC}^{*}\left[\mathrm{~A}\left(S_{\mathrm{q}}^{\mathrm{N}-1}\right)\right]$ : It is the pull back of the Chern character of the canonical generator of $\mathrm{K}^{0}(\mathbb{C})$. An element of the cyclic cohomology $\mathrm{HC}^{0}$ is a trace. The degree 0 part of the Chern character of the canonical generator of $K^{0}(\mathbb{C})$ is given by the identity map $\mathbb{C} \rightarrow \mathbb{C}$, which is trivially a trace. Pulling this back we find $\mathrm{ch}^{0}\left(\varepsilon_{\lambda}\right)=\psi_{\lambda}^{(1)}: \mathrm{A}\left(S_{\mathrm{q}}^{\mathrm{N}-1}\right) \rightarrow \mathbb{C}$ which is also a trace because it is a homomorphism to a commutative algebra. These are distinct elements of $\mathrm{HC}^{0}\left[\mathrm{~A}\left(\mathrm{~S}_{\mathrm{q}}^{\mathrm{N}-1}\right)\right]$ for different values of $\lambda$. However, because the Fredholm modules $\varepsilon_{\lambda}$ all lie in the same $\mathrm{K}$-homology class, their Chern characters are all equivalent in periodic cyclic cohomology. Indeed, applying the periodicity operator once, the cohomology classes $S\left(\psi_{\lambda}^{(1)}\right) \in \mathrm{HC}^{2}\left[\mathrm{~A}\left(\mathrm{~S}_{\mathrm{q}}^{\mathrm{N}-1}\right)\right]$ are all the same. For the computation of the pairing between K-theory and K-homology, any trace determining the same periodic cyclic cohomology class can be used. The most symmetric choice of trace is given by averaging $\psi_{\lambda}^{(1)}$ over $\lambda \in S^{1} \subset \mathbb{C}$ :

$$
\tau^{\mathrm{O}}(\mathrm{a}):=\oint_{\mathrm{S}^{1}} \psi_{\lambda}^{(1)}(\mathrm{a}) \frac{\mathrm{d} \lambda}{2 \pi i \lambda} .
$$

The result is normalized, $\tau^{0}(1)=1$, and vanishes on all the generators. The higher degree parts of $\operatorname{ch}^{*}\left(\varepsilon_{\lambda}\right)$ depend only on the K-homology class $\left[\varepsilon_{\lambda}\right]$ and can be constructed from $\tau^{0}$ by the periodicity operator.

\subsubsection{K-homology Generators for Even Spheres}

We will now construct an element $\left[\mu_{\mathrm{ev}}\right] \in \mathrm{K}^{0}\left[\mathcal{C}\left(\mathrm{S}_{\mathrm{q}}^{2 \mathrm{n}}\right)\right]$ by giving a suitable even Fredholm module $\mu:=(\mathcal{H}, F, \gamma)$.

Identify the Hilbert spaces for the representations $\psi_{ \pm}^{(2 n)}$ given in (3.7) by identifying their bases, and call this $\mathcal{H}$. The representation for the Fredholm module is

$$
\psi:=\psi_{+}^{(2 \mathfrak{n})} \oplus \psi_{-}^{(2 \mathrm{n})}
$$

acting on $\mathcal{H} \oplus \mathcal{H}$. The grading operator and the Fredholm operator are respectively,

$$
\gamma=\left(\begin{array}{cc}
1 & 0 \\
0 & -1
\end{array}\right), \quad F=\left(\begin{array}{ll}
0 & 1 \\
1 & 0
\end{array}\right) .
$$

It is obvious that $F$ is odd (since it anticommutes with $\gamma$ ) and Fredholm (since it is invertible). The remaining property to check is that for any $a \in A\left(S_{q}^{2 n}\right)$, the 
commutator $[\mathrm{F}, \psi(\mathrm{a})]_{-}$is compact. Indeed,

$$
[F, \psi(a)]_{-}=\left(\begin{array}{cc}
0 & -\psi_{+}^{(2 \mathfrak{n})}(a)+\psi_{-}^{(2 \mathfrak{n})}(\mathrm{a}) \\
\psi_{+}^{(2 \mathfrak{n})}(\mathrm{a})-\psi_{-}^{(2 \mathfrak{n})}(\mathrm{a}) & 0
\end{array}\right) .
$$

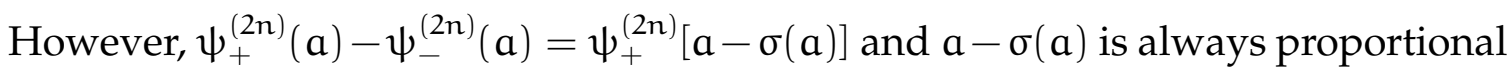
to a power of $x_{0}$. Thus this is not only compact, it is trace class. This also shows that we have (at least) a 1-summable Fredholm module. This is in contrast to the fact that the analogous element of $\mathrm{K}_{0}\left(\mathrm{~S}^{2 \mathfrak{n}}\right)$ for the undeformed sphere is given by a $2 n$-summable Fredholm module.

The Chern character [ [4] $\mathrm{ch}^{*}\left(\mu_{\mathrm{ev}}\right)$ has a component in degree $0, \operatorname{ch}^{0}\left(\mu_{\mathrm{ev}}\right) \in \mathrm{HC}^{0}\left[\mathrm{~A}\left(S_{\mathrm{q}}^{2 \mathrm{n}}\right)\right]$. The element $\operatorname{ch}^{0}\left(\mu_{\text {ev }}\right)$ is the trace

$$
\tau^{1}(a):=\operatorname{Tr}(\gamma \psi(a))=\operatorname{Tr}\left[\psi_{+}^{(2 \mathfrak{n})}(a)-\psi_{-}^{(2 \mathfrak{n})}(a)\right] .
$$

The higher degree parts of $\mathrm{ch}^{*}\left(\mu_{\mathrm{ev}}\right)$ can be obtained via the periodicity operator.

For the sphere $S_{q}^{2}$ our Fredholm module coincides with the one constructed in [14].

\subsubsection{K-homology Generators for Odd Spheres}

The element $\left[\mu_{\text {odd }}\right] \in \mathrm{K}^{1}\left[\mathcal{C}\left(\mathrm{S}_{\mathbf{q}}^{2 n+1}\right)\right]$ is most easily given by an unbounded Fredholm module.

Let the representation $\psi$ be the direct integral (over $\lambda \in S^{1}$ ) of the representations $\psi_{\lambda}^{(2 n+1)}$ given in (3.10). The operator is the unbounded operator $D:=\lambda^{-1} \frac{d}{d \lambda}$.

Referring to (3.10), we see that the representative of $x_{1}$ is proportional to $\lambda$ and as a consequence,

$$
\left[\mathrm{D}, \psi\left(\mathrm{x}_{1}\right)\right]_{-}=\psi\left(\mathrm{x}_{1}\right)
$$

whereas for $i>1$ the representative of $x_{i}$ does not involve $\lambda$ and therefore

$$
\left[\mathrm{D}, \psi\left(x_{i}\right)\right]_{-}=0, \quad i>0 .
$$

Since $a \mapsto[D, \psi(a)]_{-}$is a derivation, this shows that $[D, \psi(a)]_{-}$is bounded for any $a \in A\left(S_{q}^{2 n+1}\right)$. Note however that for $n>0$ (i. e., except for $S^{1}$ ) all eigenvalues of $D$ have infinite degeneracy and therefore $\mathrm{D}$ does not have compact resolvent.

This triple can be converted in to a bounded Fredholm module by applying a cutoff function to $D$. A convenient choice is $F=\chi(D)$ where

$$
\chi(m):= \begin{cases}1 & : m>0 \\ -1 & : m \leq 0\end{cases}
$$

To be more explicit, use a Fourier series basis for the Hilbert space,

$$
\left|k_{0}, k_{1}, \ldots, k_{n}\right\rangle:=\lambda^{k_{0}}\left|k_{1}, \ldots, k_{n}\right\rangle,
$$


in which the representation is given by,

$$
\begin{aligned}
& \psi\left(x_{1}\right)\left|k_{0}, \ldots, k_{n}\right\rangle=q^{-\left(k_{1}+\cdots+k_{n}\right)}\left|k_{0}+1, \ldots, k_{n}\right\rangle, \\
& \psi\left(x_{1}^{*}\right)\left|k_{0}, \ldots, k_{n}\right\rangle=q^{-\left(k_{1}+\cdots+k_{n}\right)}\left|k_{0}-1, \ldots, k_{n}\right\rangle, \\
& \psi\left(x_{i}\right)\left|k_{0}, \ldots, k_{n}\right\rangle=\left(1-q^{-2 k_{i-1}}\right)^{1 / 2} q^{-\left(k_{i}+\cdots+k_{n}\right)}\left|k_{0}, \ldots, k_{i-1}-1, \ldots, k_{n}\right\rangle, \\
& \psi\left(x_{i}^{*}\right)\left|k_{0}, \ldots, k_{n}\right\rangle=\left(1-q^{-2\left(k_{i-1}+1\right)}\right)^{1 / 2} q^{-\left(k_{i}+\cdots+k_{n}\right)}\left|k_{0}, \ldots, k_{i-1}+1, \ldots, k_{n}\right\rangle,
\end{aligned}
$$

for $i=1, \ldots, n$. The Fredholm operator is then given by

$$
F\left|k_{0}, \ldots, k_{n}\right\rangle=\chi\left(k_{0}\right)\left|k_{0}, \ldots, k_{n}\right\rangle .
$$

The only condition to check is that the commutator $[F, \psi(a)]_{-}$is compact for any $a \in \mathcal{C}\left(S_{\mathbf{q}}^{2 \mathfrak{n}+1}\right)$. Since $a \mapsto[F, \psi(a)]_{-}$is a derivation, it is sufficient to check this on generators. One finds $\left[F, \psi\left(x_{i}\right)\right]_{-}=0$ for $i>1$ and

$$
\left[F, \psi\left(x_{1}\right)\right]_{-}\left|k_{0}, \ldots, k_{n}\right\rangle= \begin{cases}2 q^{-\left(k_{1}+\cdots+k_{n}\right)}\left|1, k_{1}, \ldots, k_{n}\right\rangle & : k_{0}=0 \\ 0 & : k_{0} \neq 0\end{cases}
$$

which is indeed compact, and in fact trace class.

Thus, this is a 1-summable Fredholm module. Again this is in contrast to the fact that the analogous element of $K_{1}\left(S^{2 n+1}\right)$ for the undeformed sphere is given by a $(2 n+1)$-summable Fredholm module.

Its Chern character [4] begins with $\operatorname{ch}^{\frac{1}{2}}\left(\mu_{\text {odd }}\right) \in \operatorname{HC}^{1}\left[A\left(S_{\mathbf{q}}^{2 n+1}\right)\right]$ which is given by the cyclic 1-cocycle $\varphi$ defined by

$$
\varphi(\mathrm{a}, \mathrm{b}):=\frac{1}{2} \operatorname{Tr}\left(\psi(\mathrm{a})[\mathrm{F}, \psi(\mathrm{b})]_{-}\right) .
$$

The higher degree parts of $\mathrm{ch}^{*}\left(\mu_{\mathrm{odd}}\right)$ can be obtained via the periodicity operator.

For the sphere $S_{\mathrm{q}}^{3} \cong \mathrm{SU}_{\mathrm{q}}(2)$ our Fredholm module coincides with the one constructed in [13].

\subsection{K-theory for Even Spheres}

For $S_{q}^{2 n}$ we construct two classes in the $\mathrm{K}$-theory group $\mathrm{K}_{\mathrm{o}}\left[\mathcal{C}\left(\mathrm{S}_{\mathrm{q}}^{2 \mathrm{n}}\right)\right] \cong \mathbb{Z}^{2}$.

The first class is trivial. The element $[1] \in \mathrm{K}_{0}\left[\mathcal{C}\left(S_{q}^{2 n}\right)\right]$ is the equivalence class of $1 \in \mathcal{C}\left(S_{\mathbf{q}}^{2 \mathfrak{n}}\right)$ which is of course an idempotent. In order to compute the pairing with K-homology, we need the degree 0 part of its Chern character, $\mathrm{ch}_{0}[1]$, which is represented by the cyclic cycle 1 .

The second, nontrivial, class was presented in [12] It It is given by an idempotent $e_{(2 n)}$ constructed from the unipotent (2.7) as

$$
e_{(2 n)}=\frac{1}{2}\left(1+u_{(2 n)}\right) .
$$

\footnotetext{
${ }^{1}$ Again, for the sphere $S_{q}^{2}$ the idempotent (4.5) was already presented in [14].
} 
Its degree 0 Chern character, $\operatorname{ch}_{0}\left(e_{(2 n)}\right) \in H C_{0}\left[A\left(S_{q}^{2 n}\right)\right]$, is

$$
\begin{aligned}
\operatorname{ch}_{0}\left(e_{(2 \mathfrak{n})}\right)=\operatorname{tr}\left(e_{(2 \mathfrak{n})}\right) & =2^{\mathfrak{n}-1}+\frac{1}{2} \operatorname{tr}\left(\mathfrak{u}_{(2 \mathfrak{n})}\right) \\
& =2^{\mathfrak{n}-1}+\frac{1}{2}\left(q^{-1}-1\right)^{\mathfrak{n}} x_{0},
\end{aligned}
$$

since the recursive definition (2.7) of the unipotent $\mathfrak{u}_{(2 n)}$ shows that,

$$
\operatorname{tr}\left(u_{(2 n)}\right)=\left(q^{-1}-1\right) \operatorname{tr}\left(u_{(2 n-2)}\right)=\left(q^{-1}-1\right)^{n} x_{0} .
$$

Now, we can pair these classes with the two K-homology elements which we constructed in Section 4.1. First,

$$
\left\langle\varepsilon_{\lambda},[1]\right\rangle:=\tau^{0}(1)=1,
$$

which is hardly surprising. Second, the "rank" of the idempotent $e_{(2 n)}$ is

$$
\left\langle\varepsilon_{\lambda}, e_{(2 n)}\right\rangle:=\tau^{0}\left(\operatorname{ch}_{0} e_{(2 n)}\right)=2^{n-1} .
$$

Also not surprising is the "degree" of [1],

$$
\left\langle\mu_{\mathrm{ev}},[1]\right\rangle:=\tau^{1}(1)=\operatorname{Tr}\left[\psi_{+}^{(2 \mathfrak{n})}(1)-\psi_{-}^{(2 \mathfrak{n})}(1)\right]=\operatorname{Tr}(1-1)=0 .
$$

The more complicated pairing is,

$$
\begin{aligned}
\left\langle\mu_{\mathrm{ev}}, e_{(2 n)}\right\rangle: & =\tau^{1}\left(\operatorname{ch}_{0} e_{(2 n)}\right) \\
& =\operatorname{Tr} \circ \psi_{+}^{(2 n)} \circ(1-\sigma)\left(2^{n-1}+\frac{1}{2}\left[q^{-1}-1\right]^{n} x_{0}\right) \\
& =\left(q^{-1}-1\right)^{n} \operatorname{Tr}\left[\psi_{+}^{(2 n)}\left(x_{0}\right)\right] .
\end{aligned}
$$

So, we need to compute

$$
\operatorname{Tr}\left[\psi_{+}^{(2 n)}\left(x_{0}\right)\right]:=\sum_{k_{0}=0}^{\infty} \ldots \sum_{k_{n-1}=0}^{\infty} q^{-\left(k_{0}+\cdots+k_{n-1}\right)}=\left(\sum_{k=0}^{\infty} q^{-k}\right)^{n}=\left(1-q^{-1}\right)^{-n} .
$$

And, in turn, we get,

$$
\left\langle\mu_{\mathrm{ev}}, e_{(2 n)}\right\rangle=(-1)^{n} .
$$

The fact that the matrix of pairings,

\begin{tabular}{c|cc} 
& {$[1]$} & {$\left[e_{(2 \mathfrak{n})}\right]$} \\
\hline$\left[\varepsilon_{\lambda}\right]$ & 1 & 0 \\
{$\left[\mu_{\mathrm{ev}}\right]$} & 0 & $(-1)^{\mathfrak{n}}$
\end{tabular}

is invertible over the integers proves that the classes $[1],\left[e_{(2 \mathfrak{n})}\right] \in \mathrm{K}_{\mathrm{o}}\left[\mathcal{C}\left(S_{\mathbf{q}}^{2 \mathfrak{n}}\right)\right] \cong \mathbb{Z}^{2}$ and $\left[\varepsilon_{\lambda}\right],\left[\mu_{\mathrm{ev}}\right] \in \mathrm{K}^{0}\left[\mathcal{C}\left(S_{\mathrm{q}}^{2 n}\right)\right] \cong \mathbb{Z}^{2}$ are generators of these groups.

Classically, the "degree" of the left-handed spinor bundle is -1. So, the K-homology class which correctly generalizes the classical K-orientation class $[\mu] \in \mathrm{K}_{0}\left(\mathrm{~S}^{2 \mathrm{n}}\right)$ is actually $(-1)^{\mathrm{n}+1}\left[\mu_{\mathrm{ev}}\right]$. 


\subsection{K-theory for Odd Spheres}

Again, define [1] $\in \mathrm{K}_{0}\left[\mathcal{C}\left(S_{\mathrm{q}}^{2 \mathfrak{n}+1}\right)\right]$ as the equivalence class of $1 \in \mathcal{C}\left(\mathrm{S}_{\mathrm{q}}^{2 \mathfrak{n}+1}\right)$. The pairing with our element $\left[\varepsilon_{\lambda}\right] \in \mathrm{K}^{0}\left[\mathcal{C}\left(S_{\mathbf{q}}^{2 \mathfrak{n}+1}\right)\right]$ is again,

$$
\left\langle\varepsilon_{\lambda},[1]\right\rangle:=\tau^{0}(1)=1 .
$$

There is no other independent generator in $\mathrm{K}_{0}\left[\mathcal{C}\left(\mathrm{S}_{\mathrm{q}}^{2 \mathrm{n}+1}\right)\right] \cong \mathbb{Z}$.

Instead, $\mathrm{K}_{1}\left[\mathcal{C}\left(\mathrm{S}_{\mathrm{q}}^{2 \mathrm{n}+1}\right)\right] \cong \mathbb{Z}$ is nonzero. So we need to construct a generator there. An odd K-theory element is an equivalence class of unitary matrices over the algebra. We can construct an appropriate sequence of unitary matrices recursively, just as we constructed the unipotents and idempotents.

Let

$$
V_{(2 n+1)}=\left(\begin{array}{cc}
x_{n+1} & q^{-1} V_{(2 n-1)} \\
-V_{(2 n-1)}^{*} & x_{n+1}^{*}
\end{array}\right),
$$

with $V_{(1)}=x_{1}$. By using the defining relations (2.5) one directly proves that this is unitary: $\mathrm{V}_{(2 n+1)} \mathrm{V}_{(2 n+1)}^{*}=\mathrm{V}_{(2 n+1)}^{*} \mathrm{~V}_{(2 n+1)}=1$.

In order to pair our K-homology element $\left[\mu_{\text {odd }}\right] \in K^{1}\left[\mathcal{C}\left(S_{q}^{2 n+1}\right)\right]$ with the unitary $\mathrm{V}_{(2 n+1)}$, we need the lower degree part $\operatorname{ch}_{\frac{1}{2}}\left(\mathrm{~V}_{(2 n+1)}\right) \in \mathrm{HC}_{1}\left[\mathrm{~A}\left(\mathrm{~S}_{\mathrm{q}}^{2 n+1}\right)\right]$ of its Chern character. It is given by the cyclic cycle,

$$
\begin{aligned}
& \operatorname{ch}_{\frac{1}{2}}\left(V_{(2 n+1)}\right):=\frac{1}{2} \operatorname{tr}\left(V_{(2 n+1)} \otimes V_{(2 n+1)}^{*}-V_{(2 n+1)}^{*} \otimes V_{(2 n+1)}\right) \\
& =\frac{1}{2}\left(q^{-2}-1\right)^{n}\left(x_{1} \otimes x_{1}^{*}-x_{1}^{*} \otimes x_{1}\right) \text {. }
\end{aligned}
$$

Now, compute the pairing,

$$
\begin{aligned}
\left\langle\mu_{\text {odd }}, V_{(2 n+1)}\right\rangle & :=\left\langle\varphi, \operatorname{ch}_{\frac{1}{2}}\left(V_{(2 n+1)}\right)\right\rangle \\
& =-\left(q^{-2}-1\right)^{n} \varphi\left(x_{1}^{*}, x_{1}\right) \\
& =-\frac{1}{2}\left(q^{-2}-1\right)^{n} \operatorname{Tr}\left(\psi\left(x_{1}^{*}\right)\left[F, \psi\left(x_{1}\right)\right]_{-}\right) .
\end{aligned}
$$

We have already computed $\left[F, \psi\left(x_{1}\right)\right]_{-}$in eq. (4.3). From that, we get

$$
\psi\left(x_{1}^{*}\right)\left[F, \psi\left(x_{1}\right)\right]_{-}\left|k_{0}, \ldots, k_{n}\right\rangle= \begin{cases}2 q^{-2\left(k_{1}+\cdots+k_{n}\right)}\left|0, k_{1}, \ldots, k_{n}\right\rangle & : k_{0}=0 \\ 0 & : k_{0} \neq 0 .\end{cases}
$$

Thus,

$$
\begin{aligned}
\operatorname{Tr}\left(\psi\left(x_{1}^{*}\right)\left[F, \psi\left(x_{1}\right)\right]_{-}\right) & =\sum_{k_{1}=0}^{\infty} \cdots \sum_{k_{n}=0}^{\infty} 2 q^{-2\left(k_{1}+\cdots+k_{n}\right)} \\
& =2\left(\sum_{k=0}^{\infty} q^{-2 k}\right)^{n} \\
& =2\left(1-q^{-2}\right)^{-n} .
\end{aligned}
$$


Finally, this gives

$$
\left\langle\mu_{\text {odd }}, V_{(2 n+1)}\right\rangle=(-1)^{n+1} .
$$

This proves the classes $\left[\mathrm{V}_{(2 \mathrm{n}+1)}\right] \in \mathrm{K}_{1}\left[\mathcal{C}\left(S_{\mathbf{q}}^{2 n+1}\right)\right]$ and $\left[\mu_{\text {odd }}\right] \in \mathrm{K}^{1}\left[\mathcal{C}\left(S_{\mathbf{q}}^{2 n+1}\right)\right]$ are nonzero and that neither may be a multiple of another class. Thus $\left[\mathrm{V}_{(2 n+1)}\right]$ and $\left[\mu_{\text {odd }}\right]$ are indeed generators of these groups.

\section{Final remarks}

Recently, in [2], a 3-summable spectral triple was constructed for $\mathrm{SU}_{\mathrm{q}}(2)$; this has been thoroughly analyzed in [5] in the context of the noncommutative local index formula of [6]. Also, a 2-summable spectral triple on $\mathrm{SU}_{\mathrm{q}}(2)$ was constructed in [3] together with a spectral triple on the spheres $S_{\mathrm{qc}}^{2}$ of Podles̀ [16].

It would be interesting to compare our 1-summable Fredholm modules for the sphere $S_{\mathrm{q}}^{3} \cong \mathrm{SU}_{\mathrm{q}}(2)$ and for the equator sphere $S_{\mathrm{q}}^{2} \cong \mathrm{S}_{\mathrm{q} \infty}^{2}$ with these spectral triples. It seems likely that for the same spheres they determine the same K-homology class.

It would also be interesting, although much more challenging, to extend (some of) the analysis of [5] to all spheres, notably odd ones, $\mathrm{S}_{\mathrm{q}}^{2 \mathrm{n}+1}$, with a suitable modification of the unbounded Fredholm modules constructed in Section 4.1.2.

\section{Acknowledgments}

We are grateful to J. Cuntz and N. Higson for directing us to some references and to F. Bonechi, G. Fiore, L. Dabrowski, J. Madore, and D. Perrot for useful conversations. J. Varilly's remarks on the previous version have led to significant improvements.

\section{References}

[1] B. L. Cerchiai, G. Fiore, J. Madore, Geometrical tools for quantum euclidean spaces, Commun. Math. Phys. 217 (2001) 521, math.QA/0002007.

[2] P. S. Chakraborty, A. Pal, Equivariant Spectral triple on the Quantum $\mathrm{SU}(2)$-group, math.KT/0201004.

[3] P. S. Chakraborty, A. Pal, Spectral triples and associated Connes-de Rham complex for the quantum $\mathrm{SU}(2)$ and the quantum sphere, math.QA/0210049.

[4] A. Connes, Noncommutative geometry, Academic Press 1994.

[5] A. Connes, Cyclic Cohomology, Quantum group Symmetries and the Local Index Formula for $\mathrm{SU}_{\mathrm{q}}(2)$, math.QA/0209142. 
[6] A. Connes, $\mathrm{H}$. Moscovici, The local index formula in noncommutative geometry, GAFA 5 (1995) 174.

[7] J. Cuntz, On the homotopy groups for the space of endomorphisms of a $\mathrm{C}^{*}$-algebra (with applications to topological Markov chains), in Operator algebras and group representations, Pitman 1984, pp. 124-137

[8] K. R. Davidson, C*-algebras by example, American Mathematical Society 1996.

[9] L. D. Faddeev, N. Y. Reshetikhin, L. A. Takhtajan, Quantization of Lie groups and Lie algebras, Lenin. Math. Jour. 1 (1990) 193.

[10] G. Fiore, The Euclidean Hopf algebra $\mathrm{U}_{\mathrm{q}}\left(\mathrm{e}^{\mathrm{N}}\right)$ and its fundamental Hilbert space representations, J. Math. Phys. 36 (1995) 4363, hep-th/9407195.

$\mathrm{G}$. Fiore, The $q$-Euclidean algebra $\mathrm{U}_{\mathrm{q}}\left(\mathrm{e}^{\mathrm{N}}\right)$ and the corresponding $q$-Euclidean lattice, Int. J. Mod. Phys. A11 (1996) 863, q-alg/9506028.

[11] J. H. Hong, W. Szymański, Quantum spheres and projective spaces as graph algebras, University of Newcastle Preprint, January 2001; Commun. Math. Phys. in press.

[12] G. Landi, J. Madore, Twisted Configurations over Quantum Euclidean Spheres, J. Geom. Phys. in press, math.QA/0102195.

[13] T. Masuda, Y. Nakagami, J. Watanabe, Noncommutative differential geometry on the quantum SU(2). I: An algebraic viewpoint, K-Theory 4 (1990) 157.

[14] T. Masuda, Y. Nakagami, J. Watanabe, Noncommutative differential geometry on the quantum two sphere of P. Podles̀. I: An algebraic viewpoint, K-Theory 5 (1991) 151.

[15] O. Ogievetsky, Differential operators on quantum spaces for $\mathrm{GL}_{\mathrm{q}}(\mathrm{n})$ and $\mathrm{SO}_{\mathrm{q}}(\mathrm{n})$, Lett. Math. Phys. 24 (1992) 245.

[16] P. Podles̀, Quantum spheres, Lett. Math. Phys. 14 (1987) 193.

[17] I. Raeburn, W. Szymański, Cuntz-Krieger algebras of infinite graphs and matrices, University of Newcastle Preprint, December 1999.

[18] S. Sakai, C*-Algebras and $W^{*}$-Algebras, Springer 1998.

[19] L. L. Vaksman, Y. S. Soibelman, The algebra of functions on quantum $\mathrm{SU}(\mathrm{n}+1)$ group and odd-dimensional quantum spheres, Lenin. Math. Jour. 2 (1991) 1023.

[20] M. Welk, Differential Calculus on Quantum Spheres, math.QA/9802087.

[21] S. L. Woronowicz, Twisted SU(2) group. An example of a noncommutative differential calculus, Publ. Res. Inst. Math. Sci. 23 (1987) 117. 